\title{
Population-Based Regional Cancer Incidence in Korea: Comparison between Urban and Rural Areas
}

\author{
Haa-Na Song, MD \\ Se-II Go, MD' \\ Won Sup Lee, MD, PhD' \\ Yire Kim, MD' \\ Hye Jung Choi, MD 1 \\ Un Seok Lee, MD \\ Myoung Hee Kang, MD, PhD' \\ Gyeong-Won Lee, MD, PhD' \\ Hoon-Gu Kim, MD, PhD' \\ Jung Hun Kang, MD, $\mathrm{PhD}{ }^{1}$ \\ Yune Sik Kang, MD, PhD² \\ Jeong-Hee Lee, MD, $\mathrm{PhD}^{3}$ \\ Jin-Myung Jung, MD, $P h D^{4}$ \\ Soon Chan Hong, MD, PhD
}

Departments of ${ }^{1}$ Internal Medicine,

${ }^{2}$ Preventive Medicine, ${ }^{3}$ Pathology,

${ }^{4}$ Neurosurgery, and ${ }^{5}$ Surgery,

Institute of Health Sciences,

Gyeongsang National University

School of Medicine, Jinju, Korea

\section{Purpose}

The purpose of this study is to investigate differences in organ-specific cancer incidence according to the region and population size in Korea.

\section{Materials and Methods}

We reviewed the data of the cancer registration program of Gyeongnam Regional Cancer Center between 2008 and 2011. Age-standardized rates of cancer incidence were analyzed according to population size of the region and administrative zone.

\section{Results}

Incidence of thyroid cancer has been increasing rapidly in both urban and rural areas. However, the thyroid cancer incidence was much lower in rural areas than in urban areas and megalopolis such as Seoul. Gastric cancer was relatively more common in rural areas, in megalopolis near the sea (Ulsan, Busan, and Incheon), and other southern provinces (Chungcheongnam-do, Gyeongsangbuk-do, and Gyeongsangnam-do). A detailed analysis in Gyeongsangnam-do revealed that rural areas have relatively low incidence of thyroid and colorectal cancer, and relatively high incidence of gastric and lung cancer compared to urban areas.

\section{Conclusion}

This study suggests that there are some differences in cancer incidence by population size. Thyroid and colorectal cancer incidence was increasing, and gastric and lung cancer was slightly decreasing in urban areas, whereas gastric and lung cancer incidence still remains high in rural areas.

\author{
Correspondence: Won Sup Lee, MD, PhD \\ Division of Hematology-Oncology, \\ Department of Internal Medicine, \\ Gyeongsang National University \\ School of Medicine, 79 Gangnam-ro, Jinju 52727, \\ Korea \\ Tel: $82-55-750-8733$ \\ Fax: 82-55-758-9122 \\ E-mail: lwshmo@hanmail.net \\ Received February 16, 2015 \\ Accepted June 17, 2015 \\ Published Online July 14, 2015 \\ * Haa-Na Song and Se-Il Go contributed equally \\ to this work.
}

Key words

Epidemiology, Rural population, Korea, Urbanization 


\section{Introduction}

Cancer has been the leading cause of death worldwide. The mortality from cerebrovascular and cardiovascular disease decreased by 6.9 per 100,000 people during the past decade (120.4 / 100,000 people in 2001 and 113.5 / 100,000 people in 2011) [1]. However, cancer mortality increased by 19.9 per 100,000 people during that period with a steady increase in cancer incidence [1]. Cancer deaths accounted for $18 \%$ of all causes of death worldwide [2] and 27.8\% in Korea in 2011 [3].

Cancer development is significantly influenced by environmental factors. The differences in cancer incidence by geographic region were reported in previous studies.

The stomach cancer incidence of Japanese immigrants in Hawaii is lower than that of Japanese in Japan [4]. In the United States, the lung cancer incidence was highest in Kentucky and lowest in Utah; the authors found that the reason for the difference was a significant difference in smoking prevalence between the two states [5]. Given that, in addition to geographic region, population size also affects the distribution of risk factors for cancer [6,7], the relationship between population size and cancer incidence was assessed in several reports. A French study reported that the risk of colorectal cancer was $73 \%$ higher in rural patients than in urban patients after removal of the advanced adenoma [8]. In China, the incidence of primary liver cancer was higher in rural areas than in urban areas [9]. In contrast, in a study conducted in the United States, no significant difference in incidence was observed in patients with esophageal and gastric cancers, suggesting that the overall prevalence of risk factors for cancer such as obesity, smoking, alcohol consumption, and reflux disease may not differ between rural and urban areas [10].

To reduce cancer incidence and mortality, primary prevention against cancer development including life style modification and eradication of cancer risk factors, and secondary prevention such as regular health screening has been implemented in Korea. Recently, national cancer incidence data was collected by the Korea Central Cancer Registry (KCCR) program, but the regional data has not been analyzed for organ-specific cancer incidence [11]. Therefore, an analysis of cancer incidence according to population size may be helpful in determining the difference in the distribution of risk factors for cancer between rural and urban areas and in formulating an individualized health policy in each region. In this study, we investigated regional cancer incidence, focusing on the Gyeongsangnam-do regional cancer registry by collecting data from KCCR.

\section{Materials and Methods}

The KCCR cancer registration covers the entire population under the Population-Based Regional Cancer Registry Program. Details of the history, objectives, and activities of patients have been documented. We reviewed the collected age-standardized regional organ-specific cancer incidence of Gyeongsangnam-do from 2008 to 2011 from Gyeongnam Regional Cancer Center and the Korea National Cancer Incidence Database (KNCI DB). Each case of cancer was classified according to the International Classification of Diseases for Oncology third edition [12].

The proportion of the elderly population (65 years or more) was $12.5 \%$ in 2010 in Gyeongsangnam-do [3]. Given that the proportion of the elderly population is higher in rural areas than in urban areas in Korea [3], urban area was defined as a region where the proportion of that is lower than $12.5 \%$ and the remaining regions as rural area. According to this definition, regions where more than 200,000 people live were included in urban area. Urban areas were further classified into two groups based on population size: metropolitan area where more than 500,000 people live and nonmetropolitan urban area where less than 500,000 people live. Among five urban areas of Gyeongsangnam-do, metropolitan areas included Changwon and Gimhae and non-metropolitan urban areas included Jinju, Geoje, and Yangsan. The remaining 13 rural areas included Tongyeong, Sacheon, Miryang, Uiryeong, Haman, Changnyeong, Goseong, Namhae, Hadong, Sancheong, Hamyang, Geochang, and Hapcheon. Population demographics according to the region were investigated using statistical data from the Korean governmental statistics agency [3].

First, organ-specific cancer incidence in each region in Gyeongsangnam-do was analyzed. In addition, the incidences of the top five cancers were investigated: thyroid cancer, gastric cancer, colorectal cancer, hepatobiliary cancer, and lung cancer. Finally the incidences in Gyeongsangnamdo were compared with those in other megalopolises and provinces nationwide.

\section{Results}

\section{Cancer incidence in Korea and Gyeongsangnam-do}

The number of newly diagnosed cancer patients has been steadily increasing since 1999, nationwide and in Gyeongsangnam-do: the nationwide annual cancer incidence was 219.9 and 319.8 of 100,000 people in 1999 and 2011, respec- 


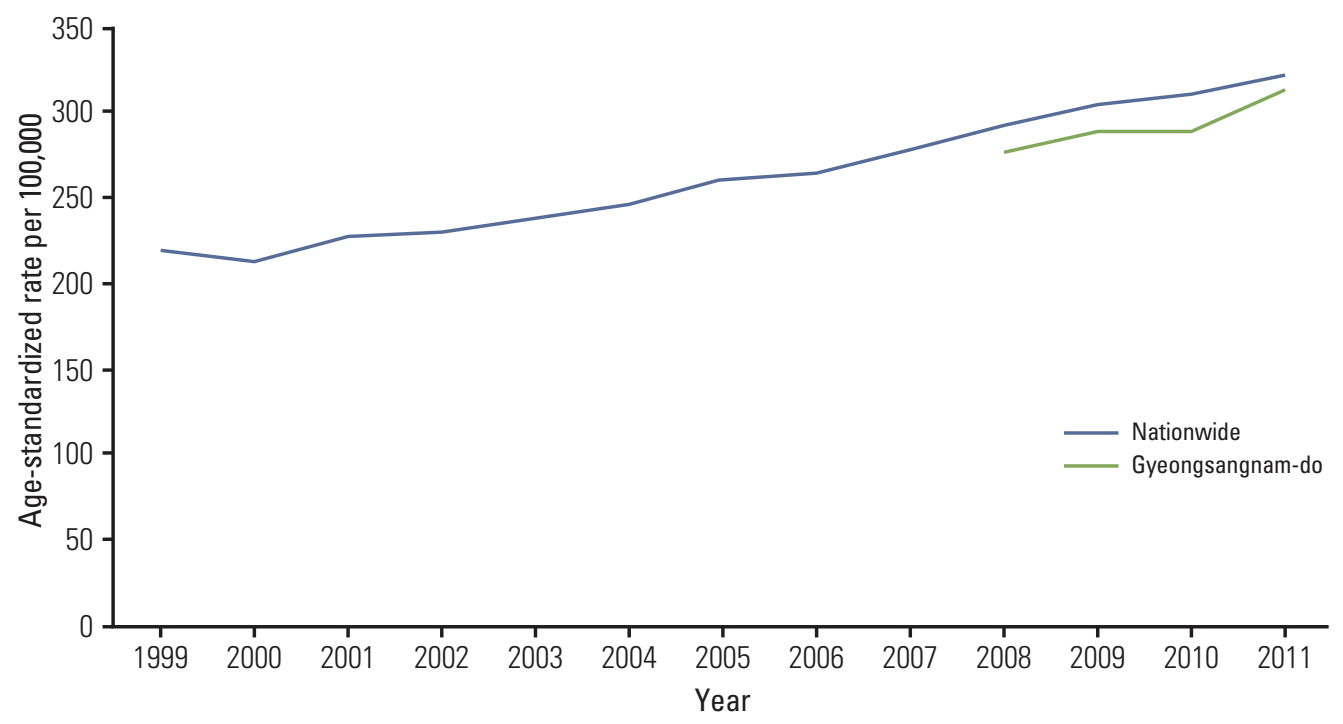

Fig. 1. Annual age-standardized cancer incidence for all sites during 1999-2011 nationwide and during 2008-2011 in Gyeongsangnam-do.

tively. The annual cancer incidence of Gyeongsangnam-do showed a similar pattern, but was somewhat lower compared with the nationwide incidence: the annual incidence of cancer patients was 276.8 and 311.5 of 100,000 people in 2008 and 2011, respectively (Fig. 1).

\section{Changes in incidences of major cancers in Korea and Gyeongsangnam-do}

Annual organ-specific cancer incidence is shown in Fig. 2. Nationwide incidence of thyroid cancer has shown a rapid increase since 2008, whereas gastric cancer, colorectal cancer, hepatobiliary cancer, and lung cancer did not. In Gyeongsangnam-do, gastric cancer was the most frequently observed malignancy before 2010, but thyroid cancer became the most common malignancy in 2011. In general, the pattern of changes in incidences of top five cancers was similar both nationwide and in Gyeongsangnam-do (Fig. 2).

\section{Incidences of major cancers by population in Gyeongsa- ngnam-do}

Population demographics by region in 2010 in Gyeongsangnam-do are presented in Table 1. By the definition described above, the proportion of the elderly population was higher in rural areas than in urban areas. The proportion of female was also higher in rural areas. As expected, higher education level and medical accessibility were observed in urban areas compared with rural areas.
The highest incidence of cancer was observed in Gimhae, and the lowest in Hamyang. The incidences of major cancers in Gyeongsangnam-do in 2011 are shown according to population size in Fig. 3. Regardless of population size, the top three cancers in Gyeongsangnam-do were thyroid cancer, gastric cancer, and hepatobiliary cancer in order of incidence. In rural areas, lung cancer and colorectal cancer were the fourth and the fifth, respectively. However, the fourth was colorectal cancer, and lung cancer was the fifth in urban areas. Incidences of thyroid cancer and colorectal cancer were higher in metropolitan areas than in non-metropolitan urban areas as well as in rural areas, whereas incidences of gastric cancer and lung cancer were highest in rural areas. The incidences of specific cancers in three areas were compared with that in Seoul, the largest city in Korea. Thyroid cancer and colorectal cancer incidences in metropolitan area of Gyeongsangnam-do were lower than those in Seoul. Hepatobiliary cancer incidence in Gyeongsangnam-do was not significantly different among three areas, but much higher than that in Seoul.

\section{Changes in incidences of major cancers by population in Gyeongsangnam-do}

Fig. 4 shows age-standardized rates for cancer incidences according to population size in Gyeongsangnam-do between 2008 and 2011. Thyroid cancer incidence increased rapidly, but the patterns differed slightly between populations. Colorectal cancer incidence appeared to be increasing with vari- 
A
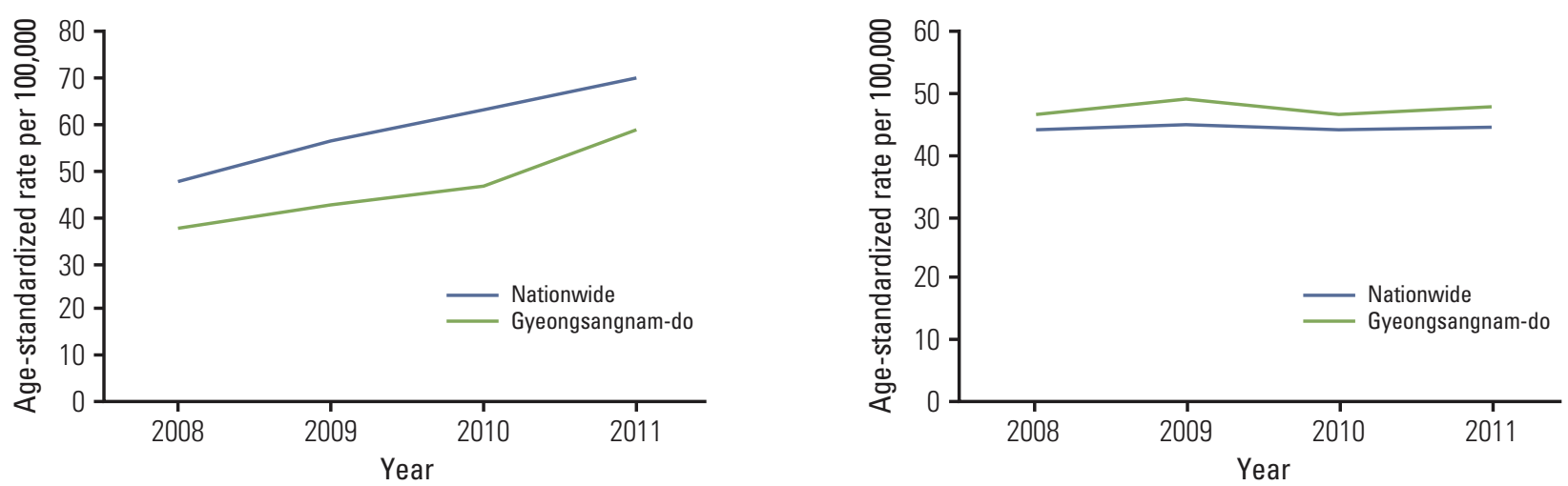

C

D
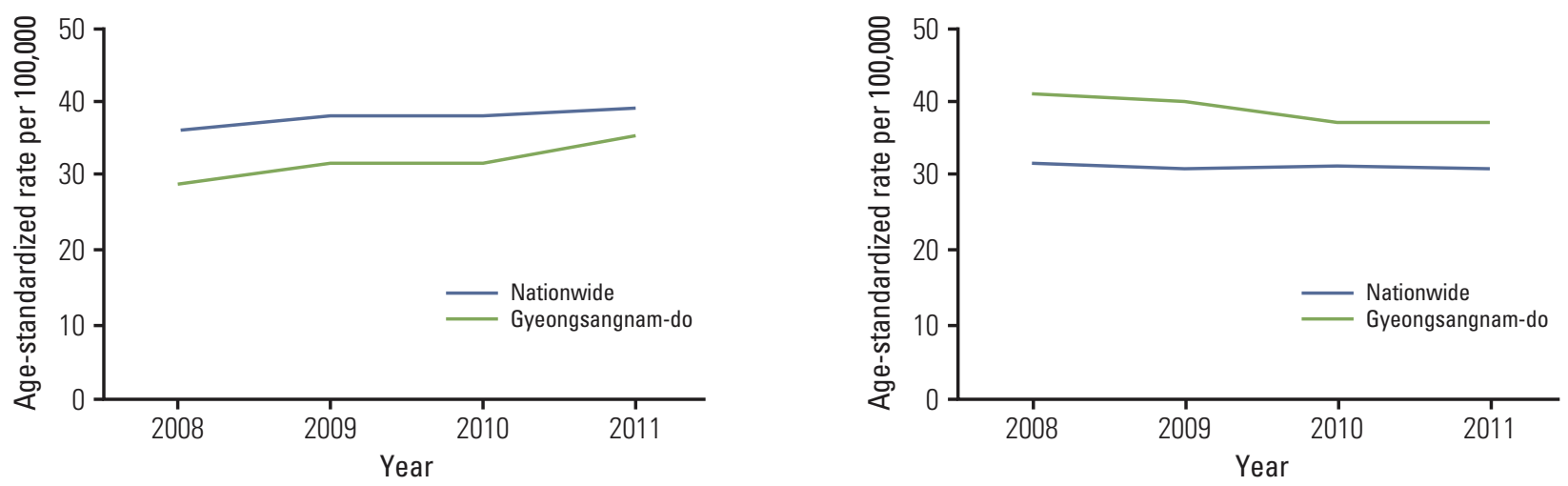

E

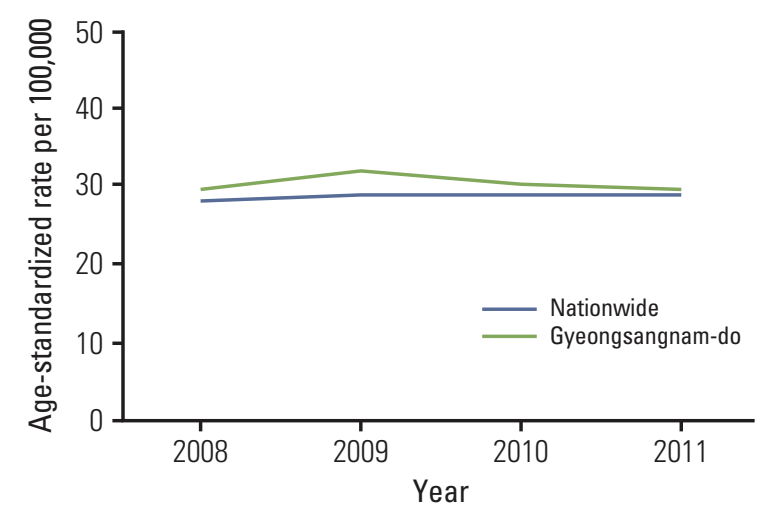

Fig. 2. Trends in age-standardized incidence of major cancers (A, thyroid cancer; B, gastric cancer; C, colorectal cancer; D, hepatobiliary cancer; and E, lung cancer) during 2008-2011 nationwide and Gyeongsangnam-do. 


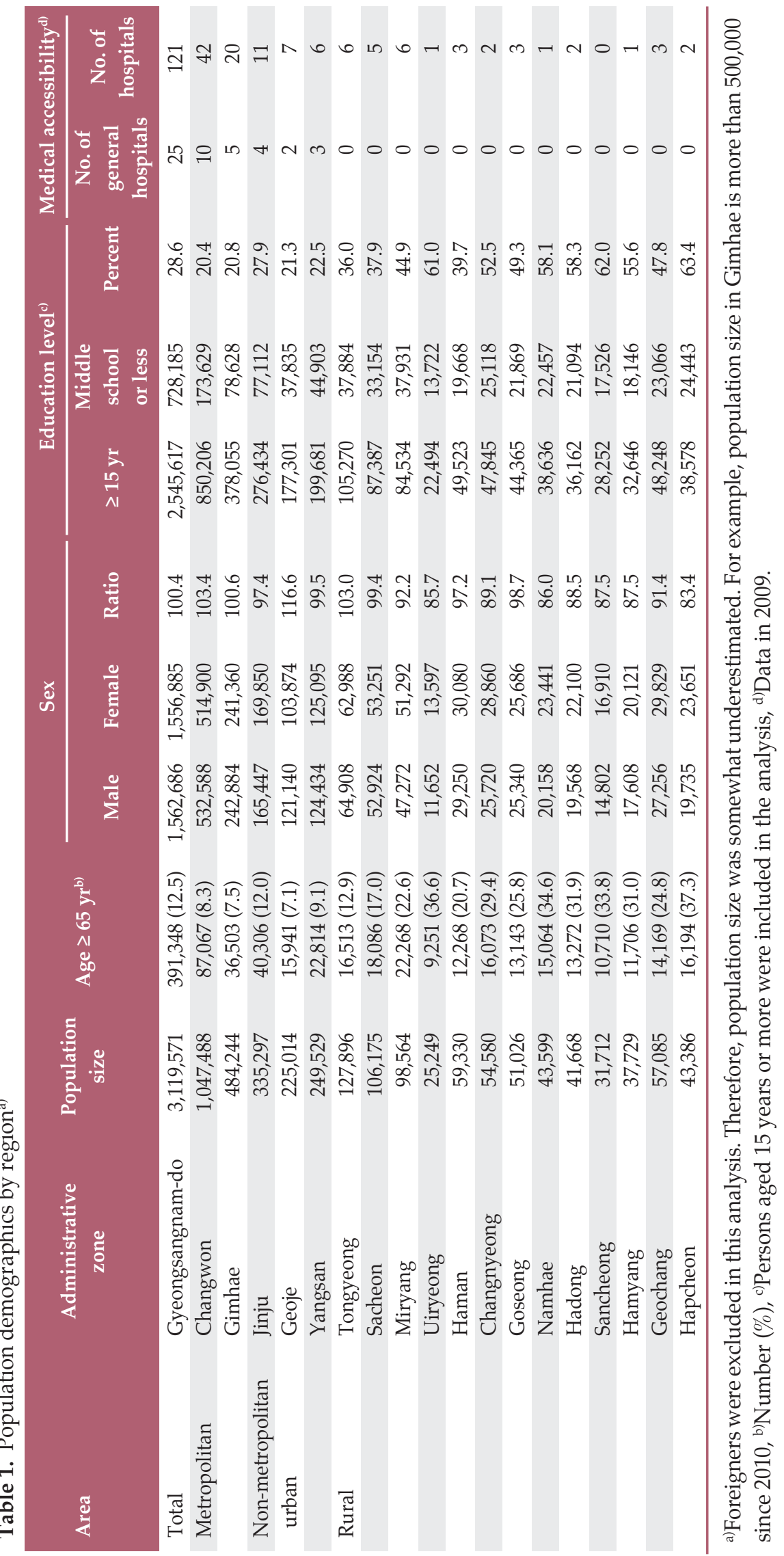




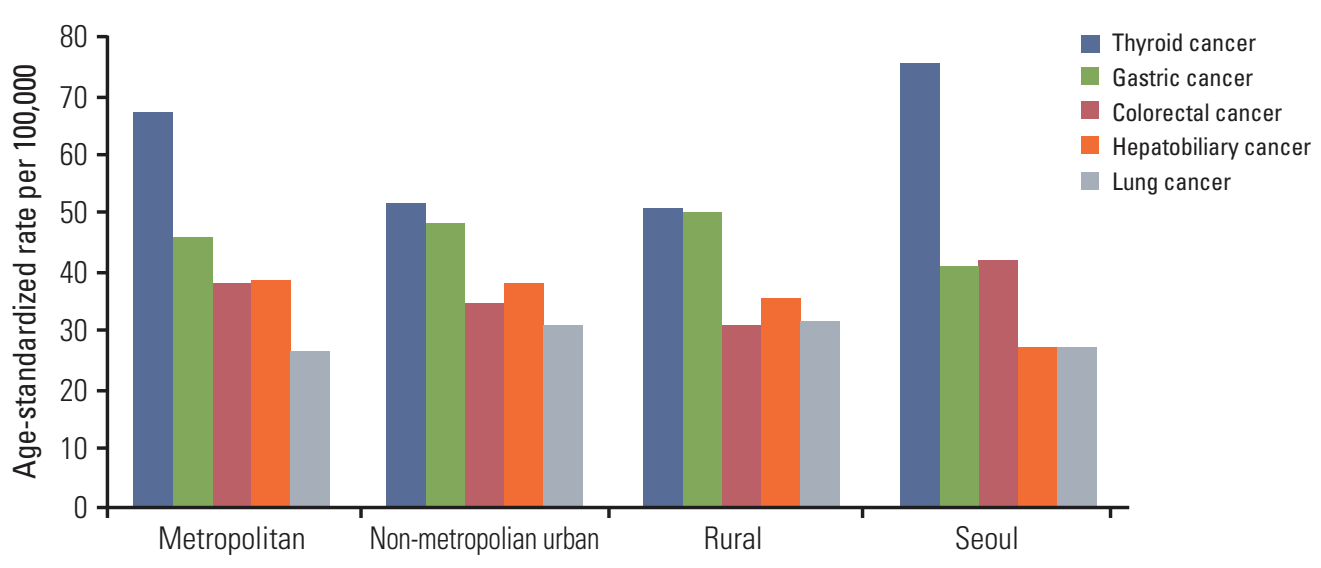

Fig. 3. Regional cancer incidence according to population size in Gyeongsangnam-do and in Seoul in 2011.

ations between years. The lung cancer incidence slightly decreased in metropolitan areas, whereas it did not change or slightly increased in non-metropolitan urban and rural areas. Hepatobiliary cancer incidence was steadily decreasing in all areas.

\section{Incidences of major cancers in megalopolises and provinces}

To validate our hypothesis, we additionally assessed the incidences of five major cancers in seven megalopolises, and five provinces (Fig. 5). Among megalopolises, the highest incidence of cancer was observed in Daegu, and the lowest in Incheon. Thyroid cancer was the most common cancer in all investigated areas. The incidences of thyroid cancer in megalopolises except Busan and Incheon were more than 70 per 100,000 person-years. In contrast, Jeollanam-do was the only province where the incidence of thyroid cancer was over 70 per 100,000 person-years. Gyeongsangnam-do had the lowest incidence of thyroid cancer among regions as shown in Fig. 5. Gastric cancer, colorectal cancer, lung cancer, and hepatobiliary cancer followed in order of incidence in most areas. However, higher incidence of hepatobiliary cancer was observed in Busan, Jeollanam-do, Gyeongsangbukdo, and Gyeongsangnam-do than in other areas. In addition, for gastric cancer, higher incidence was observed in megalopolises near the sea (Ulsan, Busan, and Incheon) than in Seoul, and southern area provinces (Chungcheongnam-do, Gyeongsangbuk-do, and Gyeongsangnam-do) showed higher incidence than did Gyeonggi-do.

\section{Discussion}

The aim of this study was to investigate regional difference in organ-specific cancer incidence by population size in Gyeongsangnam-do. Regardless of area, cancer incidence in Gyeongsangnam-do was steadily increasing, which may be attributed to two major factors. One hypothesis is the improved detection rate through health screening programs. Another hypothesis is that with advance in medical science, the elderly population with high risk for cancer development has increased. This pattern is consistent with nationwide data [11].

This study first reports that organ-specific cancer incidence differed according to population size in Korea. Regarding thyroid cancer, although its incidence was rapidly increasing in both the urban and rural areas, the incidence in rural areas was much lower than that of urban areas. Similarly, in nationwide data, incidence of thyroid cancer was generally higher in megalopolis than in other areas. Given that there is a strong correlation between the regional incidence of thyroid cancer and the proportion of the population screened in a region [13] and medical accessibility was lower in rural areas than in urban areas in our study, we assume that thyroid cancer screening was more actively conducted in urban areas than in rural areas.

The low incidence of thyroid cancer in Gyeongsangnamdo might contribute to the lower total cancer incidence, compared with the nationwide cancer incidence. We found no clear evidence to suggest why the incidence of thyroid cancer was lower in Gyeongsangnam-do than nationwide. However, among urban areas in Gyeongsangnam-do, the incidence of thyroid cancer was higher in metropolitan area than in non-metropolitan area. Therefore, relatively lower propor- 

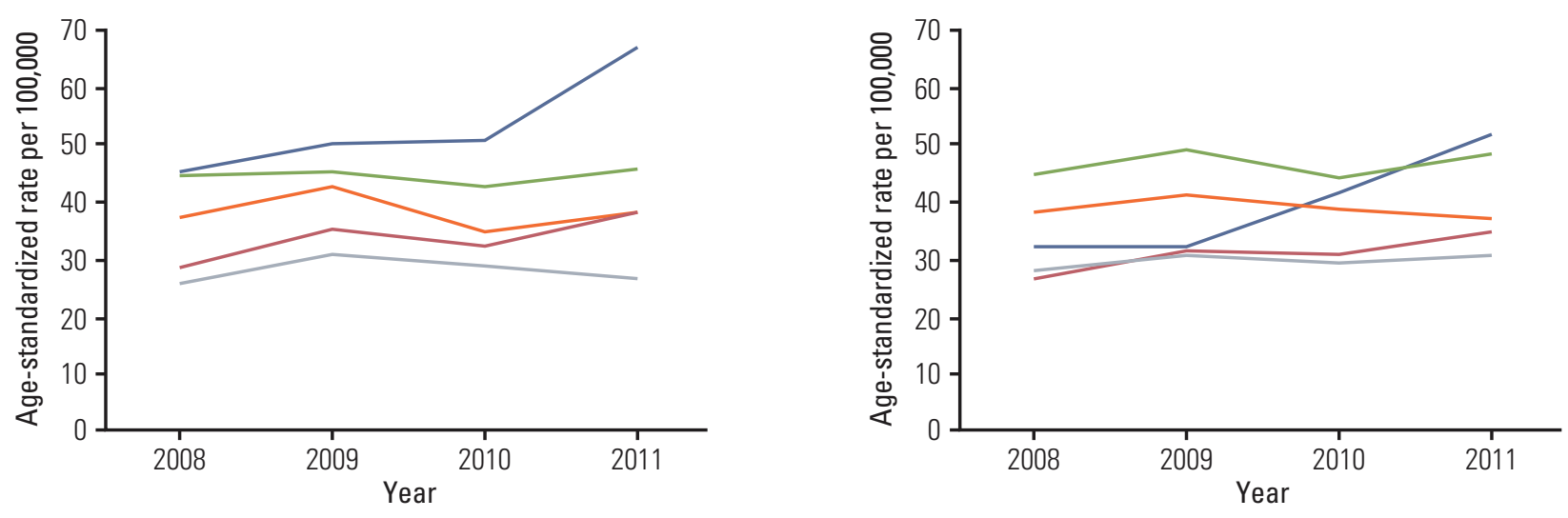

\section{C}
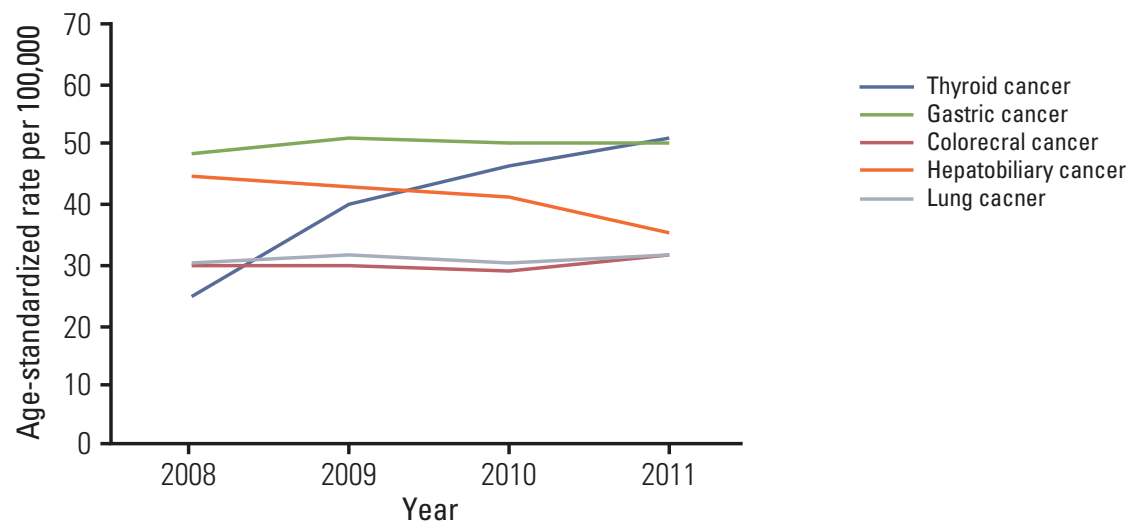

Fig. 4. Trends in age-standardized incidence of major cancers according to population size during 2008-2011 in Gyeongsangnam-do: metropolitan (A), non-metropolitan urban (B), and rural area (C).

tion of metropolitan area in Gyeongsangnam-do compared with nationwide areas may affect low incidence of thyroid cancer in Gyeongsangnam-do.

Interestingly, the thyroid cancer incidence in Busan and Incheon was lower than in other megalopolises or provinces. The two megalopolises are located near the sea and have a largesea port in common. This finding suggests that some food from the sea may have beneficial effects. The incidence of thyroid cancer depends on the food and iodine intake. This finding is supported by a previous study reported in the United States showing the differences of regional thyroid cancer incidences [14].

Second, although gastric cancer incidence has not changed in the past 10 years, its incidence was higher in the southern province than the areas near Seoul, and the incidence still remains high in rural areas. As we know, risk factors of gastric cancer development are Helicobacter pylori infection, low socioeconomic status, eating salted diets, poor hygiene, and smoking, etc. [15]. Evidence suggests that high salt intake could cause gastric cancer by direct stimulation of gastric mucosa or having a synergistic effect with $H$. pylori infection [16]. Southern areas, such as Gyeongsangnam-do and Jeollanam-do, and seaside areas, such as Busan and Incheon, showed high incidence of gastric cancer, because the population in these areas enjoys highly salted sea food sources [17]. A Japanese study reported on the relationship between gastric cancer development and amount of salt intake [18]. In another study, gastric cancer incidence was dramatically decreased by use of a refrigerator [19]. In some small rural areas, the traditional food storage methods are now being used.

Recently, the incidence of colorectal cancer is increasing in urban areas, which would be associated with westernized lifestyle, such as eating meats and fast-foods [20]. This is sup- 


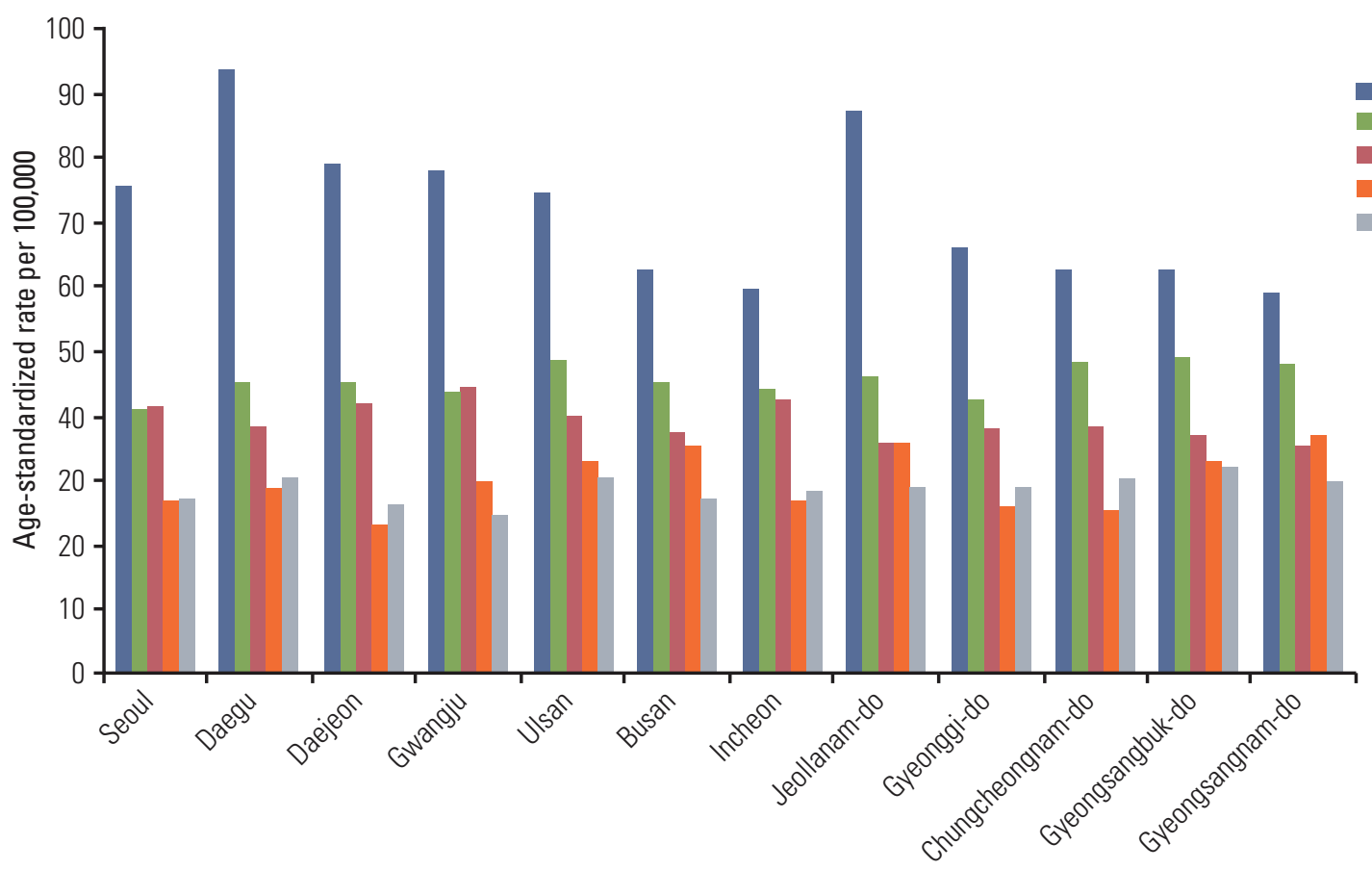

Fig. 5. Age-standardized incidence of major cancers in megalopolises and provinces in 2011.

ported by a previous study reporting that colorectal cancer incidence rates among Japanese immigrants to the United States were 4-fold higher than those of Japanese in Japan [21].

Different from colorectal cancer, the lung cancer incidence was slightly decreasing in urban areas, whereas it remains constant in rural areas. One of the major risk factors of lung cancer is smoking. The numbers of smokers in the adult population in rural areas are much higher than those in urban areas [22], and a stop smoking campaign is being conducted in the large cities for prevention of lung cancer.

Lastly, the incidence of hepatobiliary cancer in Gyeongsangnam-do and Gyeongsangbuk-do was higher than the nationwide incidence. Clonorchis sinensis infection is known as a cause of hepatobiliary cancer [23]. The infection rate was higher in the areas along the Nakdong River than in other cities [24]. This finding might explain why higher incidence of hepatobiliary cancer was observed in Gyeongsangnam-do and Gyeongsangbuk-do.

There are several limitations in this study. First, this study only investigated the regional cancer incidence obtained from the Gyeongsangnam-do cancer registration database. Therefore, we did not validate our findings in other provinces. However, in this study, by comparing the nationwide incidence, we tried to avoid bias. In addition, we supposed the reasons for the differences in regional cancer incidence by reviewing other papers. Second, because the distribution of risk factors for cancer by region was not investigated, we found no specific reason why organ-specific cancer incidence differs according to population size. As described in the introduction, instead, this study may provide information about which risk factors should be investigated in each region in future epidemiologic studies. The third limitation is that malignancies other than the top five cancers were excluded in the analysis. Therefore, further validation studies for other cancers are needed.

\section{Conclusion}

In summary, this study suggests that there are some differences in cancer incidence by population size. In urban areas, the incidences of thyroid and colorectal cancers were increasing and the incidences of gastric and lung cancers were slightly decreasing, whereas the incidences of gastric and lung cancers still remain high in rural areas. Further validation study is needed to determine the causes of the differences in cancer incidence by population size. 


\section{Conflicts of Interest}

Conflict of interest relevant to this article was not reported.

\section{Acknowledgments}

This study was supported by a grant from the National R\&D Program for Cancer Control, Ministry for Health, Welfare and Family Affairs, Republic of Korea (0820050), in part by a grant from the Korean Cancer Research Institute.

\section{References}

1. National Cancer Information Center. Cancer incidence [Internet]. Goyang: National Cancer Information Center; c2012 [cited 2012 Dec 28]. Available from: http://www.cancer.go.kr.

2. World Health Organization (WHO) Databank. WHO statistical information system [Internet]. Geneva: World Health Organization; c2013 [cited 2013 Feb 5]. Available from: http:// www.who.int/whosis.

3. Statistics Korea. Cancer mortality [Internet]. Daejeon: Korea National Statistical Office; c2011 [cited 2011 Sep 8]. Available from: http://kostat.go.kr.

4. Maskarinec G, Noh JJ. The effect of migration on cancer incidence among Japanese in Hawaii. Ethn Dis. 2004;14:431-9.

5. Siegel R, Naishadham D, Jemal A. Cancer statistics, 2012. CA Cancer J Clin. 2012;62:10-29.

6. Borders TF, Booth BM. Rural, suburban, and urban variations in alcohol consumption in the United States: findings from the National Epidemiologic Survey on Alcohol and Related Conditions. J Rural Health. 2007;23:314-21.

7. Volzke H, Neuhauser H, Moebus S, Baumert J, Berger K, Stang A, et al. Urban-rural disparities in smoking behaviour in Germany. BMC Public Health. 2006;6:146.

8. Fournel I, Cottet V, Binquet C, Jooste V, Faivre J, Bouvier AM, et al. Rural-urban differences in the long-term risk of colorectal cancer after adenoma removal: a population-based study. Dig Liver Dis. 2014;46:376-82.

9. Gao J, Xie L, Chen WQ, Zhang SW, Wu QJ, Yang Y, et al. Rural-urban, sex variations, and time trend of primary liver cancer incidence in China, 1988-2005. Eur J Cancer Prev. 2013; 22:448-54.

10. Wang Z, Goodman M, Saba N, El-Rayes BF. Incidence and prognosis of gastroesophageal cancer in rural, urban, and metropolitan areas of the United States. Cancer. 2013;119:4020-7.

11. Jung KW, Won YJ, Kong HJ, Oh CM, Lee DH, Lee JS. Cancer statistics in Korea: incidence, mortality, survival, and prevalence in 2011. Cancer Res Treat. 2014;46:109-23.

12. Fritz A, Percy C, Jack A, Shanmugaratnam K, Sobin L, Parkin $\mathrm{DM}$, et al. International classification of diseases for oncology. 3rd ed. Geneva: World Health Organization; 2000.

13. Ahn HS, Kim HJ, Welch HG. Korea's thyroid-cancer "epi- demic": screening and overdiagnosis. N Engl J Med. 2014;371: 1765-7.

14. Rose J, Wertheim BC, Guerrero MA. Regional differences in thyroid cancer presentation and survival: a SEER study. Endocr Pract. 2013;19:998-1006.

15. Piazuelo MB, Correa P. Gastric cancer: overview. Colomb Med (Cali). 2013;44:192-201.

16. Wang XQ, Terry PD, Yan H. Review of salt consumption and stomach cancer risk: epidemiological and biological evidence. World J Gastroenterol. 2009;15:2204-13.

17. D'Elia L, Rossi G, Ippolito R, Cappuccio FP, Strazzullo P. Habitual salt intake and risk of gastric cancer: a meta-analysis of prospective studies. Clin Nutr. 2012;31:489-98.

18. Shikata K, Kiyohara Y, Kubo M, Yonemoto K, Ninomiya T, Shirota $\mathrm{T}$, et al. A prospective study of dietary salt intake and gastric cancer incidence in a defined Japanese population: the Hisayama study. Int J Cancer. 2006;119:196-201.

19. La Vecchia C, Negri E, D'Avanzo B, Franceschi S. Electric refrigerator use and gastric cancer risk. Br J Cancer. 1990;62: 136-7.

20. Winkels RM, Heine-Broring RC, van Zutphen M, van HartenGerritsen S, Kok DE, van Duijnhoven FJ, et al. The COLON study: colorectal cancer: longitudinal, observational study on nutritional and lifestyle factors that may influence colorectal tumour recurrence, survival and quality of life. BMC Cancer. 2014;14:374.

21. Haenszel W, Kurihara M. Studies of Japanese migrants. I. Mortality from cancer and other diseases among Japanese in the United States. J Natl Cancer Inst. 1968;40:43-68.

22. Rybojad P, Dluski D, Rybojad B, Kedra M, Sawicki M, Skoczylas $P$, et al. Urban vs. rural patients. Differences in stage and overall survival among patients treated surgically for lung cancer. Ann Agric Environ Med. 2013;20:101-5.

23. Schwartz DA. Helminths in the induction of cancer: Opisthorchis viverrini, Clonorchis sinensis and cholangiocarcinoma. Trop Geogr Med. 1980;32:95-100.

24. Kim C, Kim HG, June KJ, Kim SY. The effects of case management for clients with clonorchiasis in riverside areas. J Korean Acad Community Health Nurs. 2012;23:427-37. 